\title{
Pulmonary artery velocity patterns in ductus arteriosus
}

\author{
NEIL WILSON, DAVID F DICKINSON, STANLEY J GOLDBERG, OLIVE SCOTT \\ From the Non-invasive Unit, Killingbeck Hospital, Leeds, and the University of Arizona, USA
}

SUMMARY In 12 patients with ductus arteriosus a characteristic velocity pattern in the pulmonary artery was identified using pulsed Doppler echocardiography. This pattern, which was absent in 52 normal control subjects and 200 consecutive patients with cardiac lesions other than ductus, consisted of a pandiastolic velocity directed away from the transducer. This pattern is thought to represent the diastolic component of flow into the pulmonary tree which occurs as a result of the left to right shunt.

Pulmonary artery velocity patterns, determined by Doppler echocardiography and analysed by time interval histography, have previously been reported for patients with ductus arteriosus. ${ }^{1-3}$ Doppler examination of a patient with ductus arteriosus is performed by imaging the main and branch pulmonary arteries from a left parasternal short axis position. Previous reports suggest that with the sample volume placed into the main pulmonary artery just proximal to the bifurcation the usual systolic velocity pattern is of flow directed away from the transducer (which we subsequently refer to as negative velocity). This negative velocity is said to be followed by disturbed flow generally directed toward the transducer (which we refer to as positively directed velocity). This diastolic pattern probably represents detection of the ductal jet. The disturbed feature, as registered by time interval histography, could be due to either alinear spectral analysis or detection of disturbed flow. One previous repor $\mathrm{t}^{2}$ mentions the presence of diastolic negatively directed velocity in the pulmonary artery, but the accepted Doppler criterion for the presence of a ductus arteriosus remains the demonstration of positively directed pulmonary artery velocity. ${ }^{3}$

In this report we describe a velocity pattern which we found in patients with ductus arteriosus and which is different from the commonly accepted pattern.

\section{Patients and methods}

The control group consisted of 52 normal subjects

Requests for reprints to Dr Neil Wilson, Killingbeck Hospital, York Road, Leeds LS14 6UQ.

Accepted for publication 24 May 1984 aged 17 days to 33 years (mean 10 years 11 months). A second control group consisted of 200 consecutively studied children with a wide spectrum of congenital cardiac disease; patients with aorticopulmonary shunts were excluded from this group. Each control subject underwent a full cross sectional echocardiographic and pulsed Doppler examination. The patient group consisted of 12 consecutive children aged 4 months to 14 years (mean 2 years 1 month), all of whom had clinical signs of ductus arteriosus. Each was examined with a commercially available pulsed Doppler echocardiograph (Honeywell Ultra-Imager) operating at a Doppler frequency of 2.25 or $3.5 \mathrm{MHz}$. The clinical diagnosis of ductus was confirmed at subsequent operation or cardiac catheterisation. A standard imaging study was performed ${ }^{4}$ to delineate intracardiac anatomy and if possible to identify the ductus. ${ }^{56}$ Subsequently, the pulmonary artery and bifurcation of the main branches were visualised in the left parasternal short axis plane, the Doppler sample volume was placed just proximal to the bifurcation, and the velocity was recorded. The main pulmonary artery was then interrogated proximally and distally in an attempt to demonstrate any other patterns.

\section{Results}

\section{CONTROL GROUPS}

In the normal control group the only systolic pulmonary artery velocity finding was a single, mainly laminar, velocity which was negatively directed. During late diastole a very small magnitude negatively directed velocity occurred just after the electrocardiographic $\mathbf{P}$ wave as previously reported. ${ }^{7}$ In the group of 200 children with forms of congenital cardiac disease no negative waveforms beginning in early 


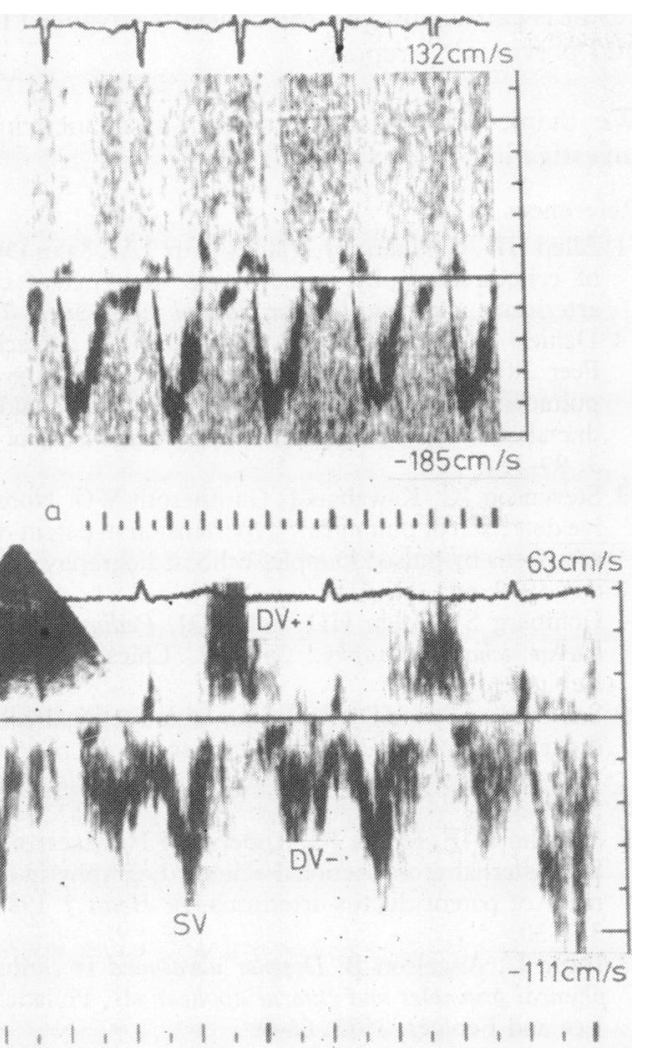

Figure (a) Normal pulmonary artery velocity pattern; single negatively directed systolic waveform. (b) Pulmonary artery velocity patterns in a patient with ductus arteriosus; positively and negatively directed diastolic waveforms $(D V+, D V-)$. $S V$, systolic waveform.

diastole were seen. The small presystolic waveform was frequently present as noted in the normal group. No other diastolic velocity findings except low level noise around the zero velocity line were noted. For the normal control group peak systolic velocities ranged from 46 to $119 \mathrm{~cm} / \mathrm{s}$ (mean $88 \mathrm{~cm} / \mathrm{s}$ ).

\section{STUDY GROUP}

In the study group the ductus was visualised in two of 12 patients. In all patients systolic velocity was negatively directed as in the control subjects. Peak systolic velocity was significantly higher $(\mathrm{p}<0.01)$ in patients with ductus arteriosus (range $70-175($ mean $=116$ ) $\mathrm{cm} / \mathrm{s}$ ) than in normal subjects. The major qualitative difference was, however, the presence of a negatively directed pandiastolic or nearly pandiastolic waveform (Figure). Peak diastolic velocity ranged from 20-120 (mean 63 ) $\mathrm{cm} / \mathrm{s}$. An additional finding in six $(50 \%)$ of the study group was a positively directed laminar or disturbed diastolic velocity pattern. This reverse velocity pattern could not be found in the other study patients.

\section{Discussion}

The most important findings of this study in patients with ductus arteriosus were $(a)$ a large magnitude negative diastolic waveform and $(b)$ an increased systolic velocity. Both findings are the direct result of ductal flow. These findings are somewhat different from, but not in conflict with, those previously reported. Previous reports of pulmonary flow characteristics, which were accomplished with alinear time interval histography spectral analysis, ${ }^{13}$ indicate that the predominant feature is a positively directed waveform in diastole. This waveform represents detection of the ductal jet. With considerable effort we were able to demonstrate this finding in half our patients. Our failure in others occurred despite optimal examination conditions since our patients were either cooperative or sedated. Although we attempted to demonstrate the positive waveform, it became clear that the predominant feature in diastole was a negatively directed waveform. We do not dispute that a jet probably exists in each of these patients, but our experience indicates that alignment of the Doppler beam with this jet is not always easy. Alignment is required in all three planes, and such alignment may not be possible in some patients because of limitations of the ultrasonic window. Furthermore, by comparison, detection of a negative directional velocity is far easier and equally specific. Neither diastolic waveform was present in the normal subjects or in the children with other conditions.

The negative directional waveform is caused by blood flow down the pulmonary tree. When a jet enters the main pulmonary artery in diastole flow occurs with the pulmonary valve closed. Diastolic flow through the ductus is consistent with the presence of the diastolic component of the continuous murmur, ${ }^{8}$ reversed aortic diastolic velocity, ${ }^{9}$ and detection of the diastolic jet. ${ }^{3}$ Since the pulmonary artery has limited distensibility, and since the diastolic flow is of high magnitude, the blood must reverse direction in the main pulmonary artery and pass down into the branches. This passage produces a diastolic negative velocity, whereas if the beam is aligned with a jet a positive directional waveform is detected. If the sample volume is placed in the post jet flow disturbance, ${ }^{10}$ disturbed flow will be detected. If the sample is placed in the area where flow has reversed a negative directional velocity will be recorded. The probability of finding each area is related to the size of the area and the direction of flow in that area. Since the 
main pulmonary artery is almost ideally located for imaging velocity along its axis it is not surprising that flow along this axis, which would be inscribed negatively, was easily detected.

The negative diastolic waveform is different in timing from the presystolic waveform found in subjects with low pulmonary artery pressure, although both have a similar generic origin. The negative diastolic waveform found in those with low pulmonary artery pressure results from atrial flow opening the pulmonary valve. This occurs almost exclusively in late diastole but on occasion can just precede the electrocardiographic $\mathbf{P}$ wave. The negative diastolic waveform in patients with a ductus is present in early diastole and is usually pandiastolic or nearly pandiastolic. Nevertheless, both waveforms probably require a low or relatively low pulmonary vascular resistance.

The magnitude of systolic velocity was modestly but significantly increased in our patients. This increase is probably due to increased systolic flow. Since the main pulmonary artery has limited distensibility any increase in flow should be accompanied by an increase in systolic velocity. Not all our patients underwent catheterisation; consequently, velocity magnitude cannot be directly related to flow magnitude in this population.

Our method of detecting the presence of a ductus arteriosus may have theoretical limitations in patients who have ductal insertion distal to the main pulmonary artery. In this group blood would flow preferentially to the left pulmonary artery. This problem, however, also applies to the method which relies on the demonstration of reverse flow in the main pulmonary artery.

Sinee completion of this study we have had the opportunity to investigate a patient with a right to left shunting ductus and severe pulmonary vascular obstructive disease. The pulmonary artery recording of this patient showed the expected negative systolic velocity, but no diastolic waveforms were present. This result is consistent with the concepts advanced in this and previous 247 reports.

We thank Gill Wharton for her assistance in this investigation.

\section{References}

1 Allen HD, Goldberg SJ, Valdes-Cruz LM, Sahn DJ. Use of echocardiography in newborns with patent ductus arteriosus: a review. Pediatr Cardiol 1982; 3: 65-70.

2 Daniels O, Hopman JCW, Stoelinga GBA, Busch HJ, Peer PGM. Doppler flow characteristics in the main pulmonary artery and the LA/Ao ratio before and after ductal closure in healthy newborns. Pediatr Cardiol 1982; 3: 99-104.

3 Stevenson JG, Kawabori I, Guntheroth WG. Noninvasive detection of pulmonary hypertension in patent ductus arteriosus by pulsed Doppler echocardiography. Circulation 1979; 60: 355-9.

4 Goldberg SJ, Allen HD, Sahn DJ. Pediatric and adolescent echocardiography. 2nd ed. Chicago: Yearbook Publisher, 1980.

5 Sahn DJ, Allen HD. Real-time cross-sectional echocardiographic imaging and measurement of the patent ductus arteriosus in infants and children. Circulation 1978; 58: 343-54.

6 Smallhorn JF, Huhta JC, Anderson RH, Macartney FJ. Suprasternal cross-sectional echocardiography in assessment of patent ductus arteriosus. Br Heart $\mathcal{F} 1982 ; 48$ : 321-30.

7 Hatle L, Angelson B. Doppler ultrasound in cardiology: physical principles and clinical applications. Philadelphia: Lea and Febiger, 1982: 66-9.

8 Serwer GA, Armstrong BE, Anderson PAW. Noninvasive detection of retrograde descending aortic flow in infants using continuous wave Doppler ultrasonography. 7 Pediatr 1980; 97: 394-400.

9 Burnard ED. A murmur that may arise from the ductus arteriosus in the human baby. Proc $R$ Soc Med 1959; 52: 77-8.

10 Kececioglu-Draelos Z, Goldberg SJ, Areias J, Sahn DJ. Verification and clinical demonstration of the echo Doppler series effect and vortex shed distance. Circulation 1981; 63: 1422-8. 